\section{Portable CRISPR-based diagnostics}

A new molecular diagnostics startup formed around CRISPR and synthetic biology technologies has raised \$35 million in a series $\mathrm{A}$, taking its total funding to $\$ 49$ million since its launch in March. The company, called Sherlock Biosciences, aims to develop affordable, instrument-free diagnostic tests for use in oncology and infectious diseases as well as industrial and agricultural applications. It is developing two platforms: SHERLOCK, which uses CRISPR to detect genetic fingerprints that signal the presence of pathogens; and INSPECTR, a synthetic biologybased tool. SHERLOCK, which stands for specific high-sensitivity enzymatic reporter unlocking, uses the RNAtargeting Cas 13 enzyme to identify specific genetic targets. Cas 13 also cleaves nearby RNAs, a 'collateral' feature useful for amplifying a reporter signal in a diagnostic test. The signal can be read with a simple paper strip or as an electrochemical readout using a mobile phone. INSPECTR, short for internal splint-pairing expression cassette translation reaction, is a DNA hybridization-based sensor that detects DNA or RNA with singlebase-pair sensitivity coupled with a bioluminescent signal, which allows visualization at room temperature. Cofounders Feng Zhang from the Broad Institute and James Collins from MIT and Harvard's Wyss Institute first published on SHERLOCK in 2017, demonstrating it could detect low levels of $\mathrm{Zika}$ virus, as well as the presence of pathogenic bacteria. Their team subsequently improved detection and signal sensitivity by combining Cas 13 with other enzymes.

Sherlock joins 2018 startup Mammoth Biosciences in the race to develop CRISPR diagnostics. Mammoth initially used a combination of Cas12 and Cas13 to simultaneously detect DNA and RNA, later adding Cas14, discovered by cofounders Jennifer Doudna and Lucas Harrington. Researchers at the University of California, San Francisco, and the Chan Zuckerberg Biohub also recently disclosed FLASH (finding low abundance sequences by hybridization), a CRISPRCas9 diagnostic for identifying drugresistant microbes.

Published online: 2 August 2019

https://doi.org/10.1038/s41587-019-0220-1

\section{Microsoft makes splash in Al-enabled lab solutions}

\author{
Microsoft unveils the first collaborations for its Station B, a platform to \\ automate lab experiments so scientists can test and reproduce ever-more- \\ complex designs.
}

M icrosoft is parlaying its machinelearning expertise into a new integrated platform for working with biological systems, and to refine it, the tech giant is partnering with a Princeton University researcher and UK-based companies Oxford Biomedica and Synthace. The platform, dubbed Station B and unveiled in March, aims to improve how life scientists go about conducting their research. It enables them first to model new biomolecules and organisms in silico, then test their designs in wet-lab experiments and finally analyze the results using machine-learning algorithms. To achieve this, the platform combines Microsoft Azure's cloud infrastructure and algorithms with Synthace's automated platform for biological experiments. For real-world testing, Microsoft has brought in one commercial enterprise, Oxford Biomedica, which will apply the platform to its genemanufacturing processes, and one academic, Princeton University molecular biologist Bonnie Bassler, who will use Station B to gain insights into bacterial biofilms. Station $\mathrm{B}$, and other solutions combining laboratory information management systems (LIMS) for uploading research data with cloudbased services for search, analysis, and discovery, promise to not only enhance reproducibility but also provide time and budgetary savings for researchers in both academia and industry.

With this move into synthetic biology, Microsoft underlines the accelerating effect that machine learning could have on understanding biological systems and designing new solutions to agricultural, industrial and therapeutic problems. This vision is what increasingly draws tech founders to synthetic biology, says John Cumbers, founder and CEO of the SynBioBeta industry group. Zymergen, of Emeryville, California, a company that uses machine learning and genomics to discover new materials and products, counts among its investors Jerry Yang, founder and former CEO of Yahoo; Ev Willams, previously chairman and CEO of Twitter; and Google's

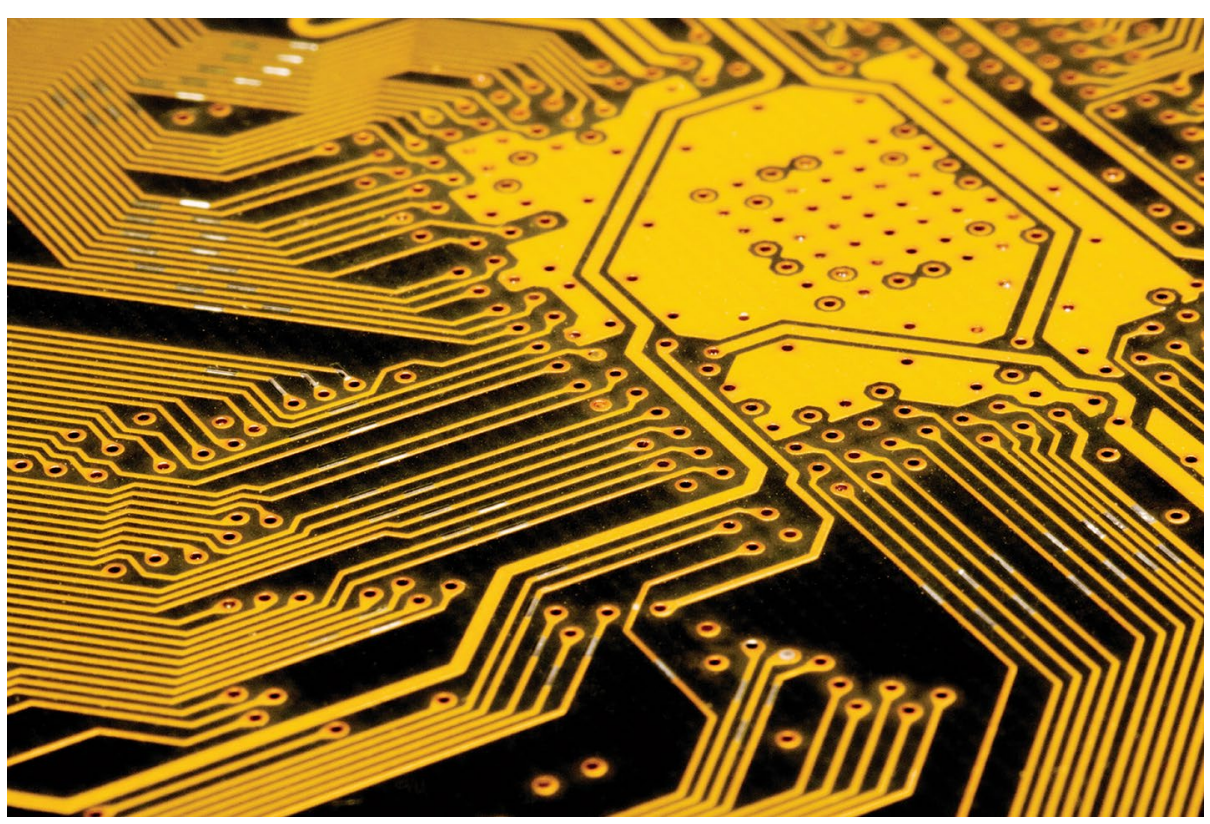

Synthetic biology researchers have started to adopt semiconductor industry processes, to create systems that ramp up throughput, boost reproducibility and ultimately reduce the costs of biopharma's increasingly complex products. Credit: South West Images Scotland / Alamy Stock Photo 